\title{
Towards testing a two-Higgs-doublet model with maximal CP symmetry at the LHC: Monte Carlo event generator implementation
}

\author{
J. Brehmer ${ }^{1, \mathrm{a}}$, V. Lendermann ${ }^{1, \mathrm{~b}}$, M. Maniatis ${ }^{2, \mathrm{c}}$, O. Nachtmann ${ }^{3, \mathrm{~d}}$, H.-C. Schultz-Coulon ${ }^{1, \mathrm{e}}$, R. Stamen ${ }^{1, \mathrm{f}}$ \\ ${ }^{1}$ Kirchhoff-Institut für Physik, Universität Heidelberg, Im Neuenheimer Feld 227, 69120 Heidelberg, Germany \\ ${ }^{2}$ Fakultät für Physik, Universität Bielefeld, Universitätsstrasse, 33615 Bielefeld, Germany \\ ${ }^{3}$ Institut für Theoretische Physik, Universität Heidelberg, Philosophenweg 16, 69120 Heidelberg, Germany
}

Received: 11 January 2013 / Revised: 23 February 2013 / Published online: 3 April 2013

(C) The Author(s) 2013. This article is published with open access at Springerlink.com

\begin{abstract}
A Monte Carlo event generator is implemented for a two-Higgs-doublet model with maximal CP symmetry, the MCPM. The model contains five physical Higgs bosons; the $\rho^{\prime}$, behaving similarly to the standard-model Higgs boson, two extra neutral bosons $h^{\prime}$ and $h^{\prime \prime}$, and a charged pair $H^{ \pm}$. The special feature of the MCPM is that, concerning the Yukawa couplings, the bosons $h^{\prime}, h^{\prime \prime}$ and $H^{ \pm}$couple directly only to the second-generation fermions but with strengths given by the third-generation-fermion masses. Our event generator allows the simulation of the Drell-Yan-type production processes of $h^{\prime}, h^{\prime \prime}$ and $H^{ \pm}$in proton-proton collisions at LHC energies. Also the subsequent leptonic decays of these bosons into the $\mu^{+} \mu^{-}, \mu^{+} \nu_{\mu}$ and $\mu^{-} \bar{v}_{\mu}$ channels are studied as well as the dominant background processes. We estimate the integrated luminosities needed in $p p$ collisions at center-of-mass energies of 8 and $14 \mathrm{TeV}$ for significant observations of the Higgs bosons $h^{\prime}, h^{\prime \prime}$ and $H^{ \pm}$in these muonic channels.
\end{abstract}

\section{Introduction}

The experimental investigation of the Higgs sector of particle physics is one of the main aims of the LHC experiments. In the standard model (SM) there is only one physical Higgs boson. But more complicated Higgs sectors are by no means excluded. In this article we present a Monte Carlo

\footnotetext{
a e-mail: johann.brehmer@kip.uni-heidelberg.de

b e-mail: victor@kip.uni-heidelberg.de

ce-mail: maniatis@physik.uni-bielefeld.de

d e-mail: o.nachtmann@thphys.uni-heidelberg.de

e e-mail: coulon@kip.uni-heidelberg.de

f e-mail: stamen@kip.uni-heidelberg.de
}

event generator for a particular two-Higgs-doublet model. The implementation of such an event generator is necessary for allowing realistic comparisons of theory and experiment to be done for this model.

Our paper is organised as follows. In Sect. 2 we recall the main construction principles and properties of the "maximally CP-symmetric model" (MCPM) which will be studied. In Sect. 3 we present predictions for the LHC experiments. In Sect. 4 the implementation of the MCPM into the Monte Carlo event-generation package MadGraph and its validation are summarized. Section 5 deals with a brief analysis of MCPM signatures using the new Monte Carlo tools. We draw our conclusions in Sect. 6.

\section{The maximally CP-symmetric model}

Extending the Standard Model Higgs sector to two Higgs doublets,

$$
\varphi_{1}=\left(\begin{array}{c}
\varphi_{1}^{+} \\
\varphi_{1}^{0}
\end{array}\right), \quad \varphi_{2}=\left(\begin{array}{c}
\varphi_{2}^{+} \\
\varphi_{2}^{0}
\end{array}\right),
$$

gives the two-Higgs-doublet model (THDM). There, the potential may contain many more terms than in the SM; see e.g. [1]. The most general THDM Higgs potential can be written as follows [2]

$$
\begin{aligned}
V= & m_{11}^{2}\left(\varphi_{1}^{\dagger} \varphi_{1}\right)+m_{22}^{2}\left(\varphi_{2}^{\dagger} \varphi_{2}\right) \\
& -m_{12}^{2}\left(\varphi_{1}^{\dagger} \varphi_{2}\right)-\left(m_{12}^{2}\right)^{*}\left(\varphi_{2}^{\dagger} \varphi_{1}\right) \\
& +\frac{1}{2} \lambda_{1}\left(\varphi_{1}^{\dagger} \varphi_{1}\right)^{2}+\frac{1}{2} \lambda_{2}\left(\varphi_{2}^{\dagger} \varphi_{2}\right)^{2}+\lambda_{3}\left(\varphi_{1}^{\dagger} \varphi_{1}\right)\left(\varphi_{2}^{\dagger} \varphi_{2}\right) \\
& +\lambda_{4}\left(\varphi_{1}^{\dagger} \varphi_{2}\right)\left(\varphi_{2}^{\dagger} \varphi_{1}\right)+\frac{1}{2}\left[\lambda_{5}\left(\varphi_{1}^{\dagger} \varphi_{2}\right)^{2}+\lambda_{5}^{*}\left(\varphi_{2}^{\dagger} \varphi_{1}\right)^{2}\right]
\end{aligned}
$$




$$
\begin{aligned}
& +\left[\lambda_{6}\left(\varphi_{1}^{\dagger} \varphi_{2}\right)+\lambda_{6}^{*}\left(\varphi_{2}^{\dagger} \varphi_{1}\right)\right]\left(\varphi_{1}^{\dagger} \varphi_{1}\right) \\
& +\left[\lambda_{7}\left(\varphi_{1}^{\dagger} \varphi_{2}\right)+\lambda_{7}^{*}\left(\varphi_{2}^{\dagger} \varphi_{1}\right)\right]\left(\varphi_{2}^{\dagger} \varphi_{2}\right)
\end{aligned}
$$

with $m_{11}^{2}, m_{22}^{2}, \lambda_{1,2,3,4}$ real and $m_{12}^{2}, \lambda_{5,6,7}$ complex. Many properties of THDMs turn out to have a simple geometric meaning if we introduce gauge invariant bilinears $[3,4]$,

$$
\begin{aligned}
& K_{0}=\varphi_{1}^{\dagger} \varphi_{1}+\varphi_{2}^{\dagger} \varphi_{2}, \\
& \mathbf{K}=\left(\begin{array}{c}
K_{1} \\
K_{2} \\
K_{3}
\end{array}\right)=\left(\begin{array}{c}
\varphi_{1}^{\dagger} \varphi_{2}+\varphi_{2}^{\dagger} \varphi_{1} \\
i \varphi_{2}^{\dagger} \varphi_{1}-i \varphi_{1}^{\dagger} \varphi_{2} \\
\varphi_{1}^{\dagger} \varphi_{1}-\varphi_{2}^{\dagger} \varphi_{2}
\end{array}\right) .
\end{aligned}
$$

In terms of these bilinears $K_{0}, \mathbf{K}$, the Higgs potential (2) reads

$$
V=\xi_{0} K_{0}+\xi^{\mathrm{T}} \mathbf{K}+\eta_{00} K_{0}^{2}+2 K_{0} \eta^{\mathrm{T}} \mathbf{K}+\mathbf{K}^{\mathrm{T}} E \mathbf{K}
$$

with parameters $\xi_{0}, \eta_{00}, 3$-component vectors $\xi, \eta$ and a $3 \times 3$ matrix $E=E^{\mathrm{T}}$, all real.

The standard $\mathrm{CP}$ transformation of the Higgs-doublet fields is defined by

$\varphi_{i}(x) \rightarrow \varphi_{i}^{*}\left(x^{\prime}\right), \quad i=1,2 x^{\prime}=\left(x^{0},-\mathbf{x}\right)$.

In terms of the bilinears, this standard $\mathrm{CP}$ transformation is $[5,6]$

$$
K_{0}(x) \rightarrow K_{0}\left(x^{\prime}\right), \quad \mathbf{K}(x) \rightarrow \bar{R}_{2} \mathbf{K}\left(x^{\prime}\right)
$$

where $\bar{R}_{2}=\operatorname{diag}(1,-1,1)$, corresponding in $K$ space to a reflection on the 1-3 plane. Generalised CP transformations (GCPs) are defined by [7-9]

$\varphi_{i}(x) \rightarrow U_{i j} \varphi_{j}^{*}\left(x^{\prime}\right), \quad i, j=1,2$,

with $U$ being an arbitrary unitary $2 \times 2$ matrix. In terms of the bilinears this reads [6]

$$
K_{0}(x) \rightarrow K_{0}\left(x^{\prime}\right), \quad \mathbf{K}(x) \rightarrow \bar{R} \mathbf{K}\left(x^{\prime}\right)
$$

with an improper rotation matrix $\bar{R}$.

Requiring $\bar{R}^{2}=\mathbb{1}_{3}$ leads to two types of GCPs. In $K$ space:

(i) $\bar{R}=-\mathbb{1}_{3}$, point reflection,

(ii) $\quad \bar{R}=R^{\mathrm{T}} \bar{R}_{2} R, \quad$ reflection on a plane $(R \in S O(3))$.

For a review of the bilinear formalism and its relation to the conventional field approach as well as the generalised $\mathrm{CP}$ transformations we refer to [10]. There, also an extensive list of references is given.
While the CP transformations of type (ii) in (10) are equivalent to the standard $\mathrm{CP}$ transformation (5), respectively (6), up to a basis change, the point reflection transformation of type (i) is quite different and turns out to have very interesting properties. Motivated by this geometric picture of generalised CP transformations, the most general THDM invariant under the point reflection (i) has been studied in [11-14]. Invariance of the THDM potential (4) under the GCP transformation (8) with $\bar{R}=-\mathbb{1}_{3}$ from (9) clearly requires $\xi=\eta=0$, leading to

$V_{\mathrm{MCPM}}=\xi_{0} K_{0}+\eta_{00} K_{0}^{2}+\mathbf{K}^{\mathrm{T}} E \mathbf{K}$.

Without loss of generality the $3 \times 3$ matrix $E$ can be chosen to be diagonal

$E=\operatorname{diag}\left(\mu_{1}, \mu_{2}, \mu_{3}\right)$

with the ordering

$\mu_{1} \geq \mu_{2} \geq \mu_{3}$.

The conditions to obtain a physically acceptable theory are spelled out in [11, 12] and read

$\eta_{00}>0$,

$\mu_{a}+\eta_{00}>0, \quad$ for $a=1,2,3$,

$\xi_{0}<0$,

$\mu_{3}<0$.

The potential (11) of this model is, besides the point reflection symmetry of type (i), invariant under three GCPs of type (ii); see (9), (10). Requiring also the Yukawa couplings to respect these four GCPs, it was found in [11] that at least two fermion families are necessary in order to have non-vanishing fermion masses. That is, a reason for family replication was given. For two fermion families only three options for the Yukawa-coupling structure were found:

(a) one massive and one massless family,

(b) two mass-degenerate families,

(c) large flavour changing neutral currents.

The maximally CP-symmetric model (MCPM) was constructed as follows in $[11,12]$. The option (a) above was chosen for the Yukawa couplings with the third family $(t, b, \tau)$ as massive one, the second family $(c, s, \mu)$ as massless one. The first family $(u, d, e)$ was added uncoupled to the Higgs fields. In this way the highly symmetric MCPM gives a first approximation to the fundamental mass spectrum as observed in nature; see Sect. 5 of [11]. The Yukawa couplings of the MCPM read 


$$
\begin{aligned}
\mathcal{L}_{\mathrm{Yuk}}(x)= & -c_{l 3}^{(1)}\left\{\bar{\tau}_{R}(x) \varphi_{1}^{\dagger}(x)\left(\begin{array}{c}
v_{\tau L}(x) \\
\tau_{L}(x)
\end{array}\right)\right. \\
& \left.-\bar{\mu}_{R}(x) \varphi_{2}^{\dagger}(x)\left(\begin{array}{c}
v_{\mu L}(x) \\
\mu_{L}(x)
\end{array}\right)\right\} \\
& +c_{u 3}^{(1)}\left\{\bar{t}_{R}(x) \varphi_{1}^{\mathrm{T}}(x) \epsilon\left(\begin{array}{c}
t_{L}(x) \\
b_{L}(x)
\end{array}\right)\right. \\
& \left.-\bar{c}_{R}(x) \varphi_{2}^{\mathrm{T}}(x) \epsilon\left(\begin{array}{c}
c_{L}(x) \\
s_{L}(x)
\end{array}\right)\right\} \\
& -c_{d 3}^{(1)}\left\{\bar{b}_{R}(x) \varphi_{1}^{\dagger}(x)\left(\begin{array}{l}
t_{L}(x) \\
b_{L}(x)
\end{array}\right)\right. \\
& \left.-\bar{s}_{R}(x) \varphi_{2}^{\dagger}(x)\left(\begin{array}{c}
c_{L}(x) \\
s_{L}(x)
\end{array}\right)\right\}
\end{aligned}
$$

where $c_{l 3}^{(1)}, c_{u 3}^{(1)}$ and $c_{d 3}^{(1)}$ are real positive constants, determined by the fermion masses as discussed below.

In the unitary gauge electroweak symmetry breaking (EWSB) gives

$$
\begin{aligned}
\varphi_{1}(x) & =\frac{1}{\sqrt{2}}\left(\begin{array}{c}
0 \\
v_{0}+\rho^{\prime}(x)
\end{array}\right), \\
\varphi_{2}(x) & =\left(\begin{array}{c}
H^{+}(x) \\
\frac{1}{\sqrt{2}}\left(h^{\prime}(x)+i h^{\prime \prime}(x)\right)
\end{array}\right)
\end{aligned}
$$

with the standard vacuum expectation value

$$
v_{0}=\sqrt{\frac{-\xi_{0}}{\eta_{00}+\mu_{3}}} \approx 246 \mathrm{GeV}
$$

The physical Higgs-boson fields are $\rho^{\prime}, h^{\prime}, h^{\prime \prime}, H^{+}$, and $H^{-}=\left(H^{+}\right)^{\dagger}$. Their masses-at tree level-read $[11,12]$

$m_{\rho^{\prime}}^{2}=2\left(-\xi_{0}\right)$,

$m_{h^{\prime}}^{2}=2 v_{0}^{2}\left(\mu_{1}-\mu_{3}\right)$,

$m_{h^{\prime \prime}}^{2}=2 v_{0}^{2}\left(\mu_{2}-\mu_{3}\right)$,

$m_{H^{ \pm}}^{2}=2 v_{0}^{2}\left(-\mu_{3}\right)$

where the ordering of the masses

$m_{h^{\prime}}^{2} \geq m_{h^{\prime \prime}}^{2}$

is predicted by the theory; see (13). Requiring now that the neutral Higgs bosons $h^{\prime}$ and $h^{\prime \prime}$ are neither massless nor mass degenerate leads to the condition

$\mu_{1}>\mu_{2}>\mu_{3}$, replacing the weaker one in (13). The masses of $\tau, t, b$ are given by

$$
\begin{aligned}
& m_{\tau}=c_{l 3}^{(1)} \frac{v_{0}}{\sqrt{2}}, \\
& m_{t}=c_{u 3}^{(1)} \frac{v_{0}}{\sqrt{2}}, \\
& m_{b}=c_{d 3}^{(1)} \frac{v_{0}}{\sqrt{2}} .
\end{aligned}
$$

This fixes the constants of the Yukawa couplings in (15) in terms of the third-family fermion masses.

The complete Lagrangian after EWSB is given in Appendix A of [12] and is implemented as Monte Carlo program for the Feynman rules in Appendix of the present paper.

In total, there are 11 parameters in the MCPM Lagrangian. Before EWSB the natural parameters are the gauge couplings $g, g^{\prime}, g_{s}$, the Higgs potential parameters $\mu_{1}, \mu_{2}, \mu_{3}, \xi_{0}, \eta_{00}$, and the Yukawa coefficients $c_{l 3}^{(1)}, c_{u 3}^{(1)}$, $c_{d 3}^{(1)}$. It is convenient to replace the above 11 parameters by another set of 11 independent parameters containing more directly and well measurable quantities. These are the electromagnetic and strong coupling constants $\alpha$ and $\alpha_{s}$, respectively, the Fermi constant $G_{F}$, the $Z$-boson mass $m_{Z}$, the fermion masses $m_{t}, m_{b}, m_{\tau}$, and the Higgs boson masses $m_{\rho^{\prime}}, m_{h^{\prime}}, m_{h^{\prime \prime}}, m_{H^{ \pm}}$. The relation of these 11 parameters to the original ones is given in Appendix. The masses of the physical Higgs bosons are constrained by (19) and from the experimental results on the so called oblique parameters in electroweak precision tests; see [15].

Let us summarize the essential properties of the MCPM:

- There are 5 physical Higgs particles, two CP even ones $\rho^{\prime}, h^{\prime}$, one CP odd one $h^{\prime \prime}$, and a charged Higgsboson pair $H^{ \pm}$.

- The $\rho^{\prime}$ boson's fermionic couplings are exclusively to the third $(\tau, t, b)$ family. The $\rho^{\prime}$ behaves similarly to the SM Higgs boson.

- The Higgs-boson-fermion couplings of $h^{\prime}, h^{\prime \prime}, H^{ \pm}$are exclusively to the second $(\mu, c, s)$ family with strengths proportional to the masses of the third-generation fermions.

- The first $(e, u, d)$ family is uncoupled to the Higgs bosons.

For further details we refer to [11-15].

\section{Predictions for hadron colliders}

Since the Yukawa couplings of the $h^{\prime}, h^{\prime \prime}, H^{ \pm}$Higgs bosons to the second fermion family are proportional to the thirdfermion-family masses we have for them large cross sections for Drell-Yan type production. For the same reason 


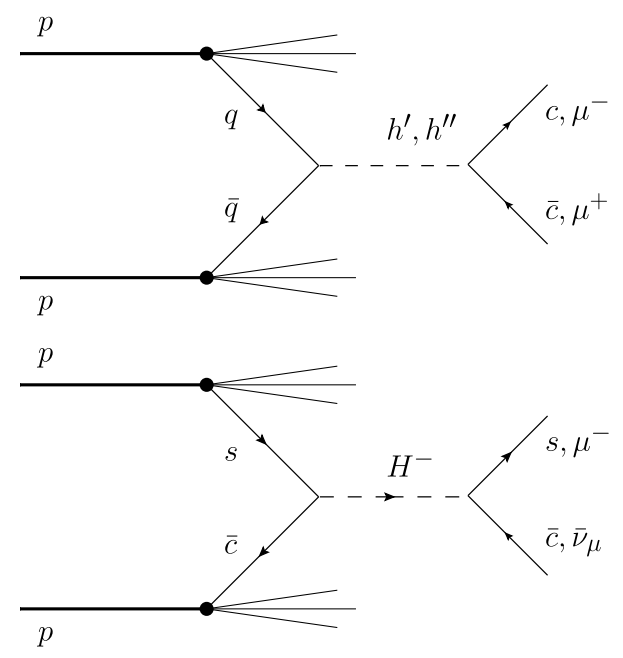

Fig. 1 Feynman diagrams for the dominant Drell-Yan type production processes for the Higgs bosons $h^{\prime}, h^{\prime \prime}$ and $H^{-}$in the MCPM $(q=c, s)$. The dominant hadronic and leptonic decays are also indicated

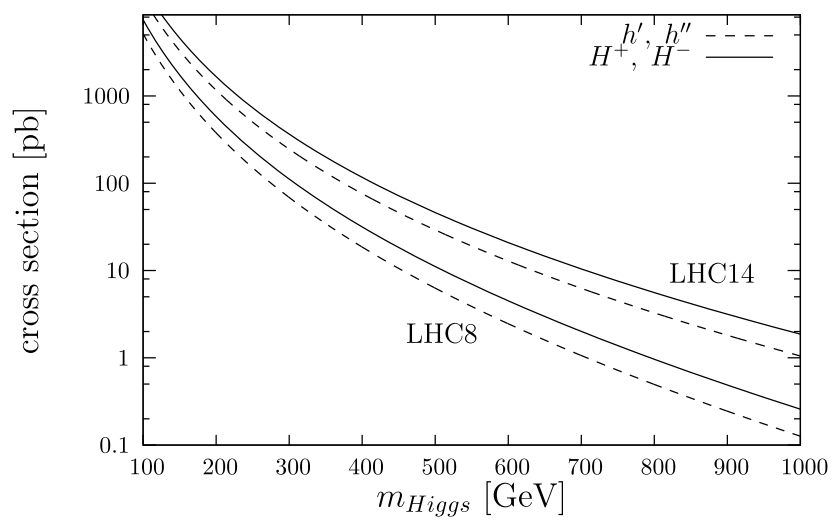

Fig. 2 Total cross section of Drell-Yan type Higgs boson production at the $\mathrm{LHC}$ for $\mathrm{c}$. $\mathrm{m}$. energies of 8 and $14 \mathrm{TeV}$, respectively

we have large decay rates of these Higgs bosons to the second generation fermions. In Fig. 1 we show the leading order diagrams for these production and decay reactions for the $h^{\prime}, h^{\prime \prime}$, and $H^{-}$bosons in $p p$ collisions. The corresponding diagram for $\mathrm{H}^{+}$production and decay is similar to the lower one of Fig. 1 with the replacements $H^{-} \rightarrow H^{+}$, $s \rightarrow c, \bar{c} \rightarrow \bar{s}, \mu^{-} \rightarrow v_{\mu}$ and $\bar{v}_{\mu} \rightarrow \mu^{+}$. In $[12,14]$ the cross sections were computed for Drell-Yan Higgs-boson production at the LHC for center-of-mass energies of 7 and $14 \mathrm{TeV}$, respectively. In [13] radiative effects were considered. Here, we add the cross sections for a center-of-mass energy of $8 \mathrm{TeV}$ which is currently available at the LHC. The corresponding total cross sections for the Drell-Yan production of the $h^{\prime}, h^{\prime \prime}, H^{ \pm}$bosons are shown in Fig. 2. In Fig. 3 we also recall the branching ratios of the $h^{\prime \prime}$ boson decays. In the following we shall develop all the necessary theoretical tools for detailed comparisons of the MCPM predictions with experimental data at the LHC. In particular,

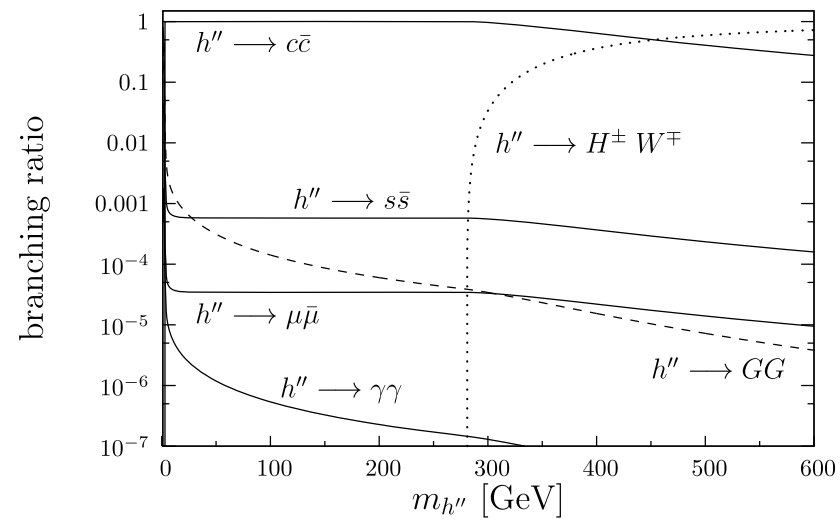

Fig. 3 Branching ratios of the CP odd $h^{\prime \prime}$ Higgs boson, where a mass of $m_{H^{ \pm}}=200 \mathrm{GeV}$ is assumed. Figure taken from [12]

we describe the implementation of the MCPM into a Monte Carlo event generator.

In the present paper we only consider $h^{\prime}, h^{\prime \prime}$ and $H^{ \pm}$production to leading order in the strong coupling parameter $\alpha_{s}$. Of course, the next step would be to include higher order QCD corrections where further production processes come into play. For instance, at parton level we have then also

$c g \rightarrow h^{\prime} c$,

$\bar{c} g \rightarrow h^{\prime} \bar{c}$,

$c \bar{c} \rightarrow h^{\prime} g$

and similarly for $h^{\prime \prime}$. We make some remarks on such processes in Sect. 5 below. But we emphasize that a complete calculation of QCD corrections for the production of our Higgs bosons is beyond the scope of the present article.

\section{Implementation of Monte Carlo event generation}

We have implemented the MCPM into the Monte Carlo event-generation package MadGraph 5/MadEvent and validated this implementation with different methods.

MadGraph 5 [16] is the latest version of the matrixelement generator MadGraph. It is bundled with the eventgeneration package MadEvent. For simplicity, both will be referred to as MadGraph in the following. Given an arbitrary process for any implemented model, the program lists all contributing tree-level diagrams. For each of these diagrams, events are randomly produced and the cross section or decay width is calculated. The output of such a simulation is a list of events in the Les Houches Event File (LHEF) standard [17]. For each event it includes the particles involved and their four-momenta. This can be read by programs such as PYTHIA [18], with which hadronisation effects can be simulated.

MadGraph is a flexible framework and allows the implementation of new models through the Universal FeynRules 
Output (UFO) format [19]. Its major downside is the limitation to tree-level processes. However, the most interesting and relevant processes in the MCPM do not involve any loops [12], so this drawback is acceptable for our present study.

The implementation includes all particles and couplings of the MCPM. This not only allows the analysis of signal processes involving Higgs bosons, but also the simulation of any tree-level background process. The quantities listed at the end of Sect. 2, namely

$\alpha, G_{F}, \alpha_{s}, m_{Z}, m_{t}, m_{b}, m_{\tau}, m_{\rho^{\prime}}, m_{h^{\prime}}, m_{h^{\prime \prime}}$, and $m_{H^{ \pm}}$,

are used as independent parameters.

In addition, more parameters can be used for the study of SM background processes. At tree level the MCPM predicts massless fermions for the first and second generation and a unit CKM matrix, $V_{\mathrm{CKM}}=\mathbb{1}_{3}$. Of course, this is not what we observe in nature, but it may represent a first approximation. Indeed, the ratios of the masses of first and second generation fermions to the corresponding third generation ones are small and $V_{\mathrm{CKM}}$ is close to the unit matrix; see (125) and (126) of [11]. It may thus be that the GCPs of the MCPM play the role of approximate symmetries. Thus, it should be sensible to analyse a model that combines the scalar sector of the MCPM with fermions of non-zero mass and a CKM matrix $V_{\mathrm{CKM}} \neq \mathbb{1}_{3}$, as done already in [12].

For this reason our MadGraph implementation of the MCPM lets the user set fermion masses for the first two families as well. This affects phase space calculations, but the interactions remain unchanged. In a similar way the CKM matrix can be set to an arbitrary matrix, which changes SM processes, but not the Higgs boson vertices. With these parameters the user can decide whether to analyse the strict MCPM or a hybrid theory with fermion masses and a SMlike CKM matrix.

The MadGraph implementation of the MCPM was validated in two different ways. First, total cross sections and decay widths were checked for a number of different processes and parameter sets. These include the production of the MCPM Higgs bosons via quark-antiquark fusion as well as their fermionic decays. In all processes and parameter configurations good agreement between Monte Carlo results and theoretical expectation was found.

Second, angular dependencies and invariant mass distributions were analysed for different processes including the Drell-Yan-type production of Higgs bosons followed by their decay into fermion pairs. Again, the event shapes agree well with the expected distributions. For more details see [20].

\section{Analysis of MCPM signatures}

It is now straightforward to ask whether the MCPM can be discovered or excluded at the LHC. A full answer requires a thorough analysis including the simulation of fragmentation and detector behaviour, which goes beyond the scope of this publication. The hadronic decay modes will thus not be considered here. A data analysis would neccessiate the tagging of charm jets which is experimentally challenging in the presence of huge QCD backgrounds. A study using a parton-level MC generator would not yield sensible results.

However, there is no hadronisation for leptons. Muons, which play an important role in the MCPM can be reconstructed relatively precisely in experiments. Hence, for the muonic decay channels the hard-process events generated by MadGraph are worth a look even without fragmentation and detector simulation. As a first application we use the new MadGraph implementation to compare the muonic MCPM signatures to the SM background.

Two such channels are analysed. The first one consists of the production of $h^{\prime}$ or $h^{\prime \prime}$ via quark-antiquark fusion with subsequent decay into a $\mu^{-} \mu^{+}$pair. The dominant SM background is given by the $\gamma^{*}$ and $Z$ Drell-Yan processes. As second channel we analyse the production of a $H^{-}$boson decaying into a $\mu^{-} \bar{v}_{\mu}$ final state. Here the dominant background stems from the production of $W^{-}$bosons. The corresponding Feynman diagrams can be found in Fig. 1. Additional backgrounds from e.g. top production have not been considered. Experimental studies show that they are below $15 \%$ for most of the tested phase space [21-24]. The production and decay of $\mathrm{H}^{+}$bosons is analogous to $\mathrm{H}^{-}$bosons and could be analysed in the same way.

Signal and background processes are simulated separately. Due to the different helicity structure of the lepton pairs from gauge-boson decays on the one hand and from Higgs-boson decays on the other hand there is, neglecting the lepton masses, no interference of signal and background. The couplings of $\mu^{+} \mu^{-}$to $\gamma^{*}$ and $Z$ are chirality conserving. Thus, in the limit $m_{\mu}=0$ only the helicity combinations $\left(h_{+}, h_{-}\right)=\left(\frac{1}{2},-\frac{1}{2}\right)$ and $\left(-\frac{1}{2}, \frac{1}{2}\right)$ occur for the $\mu^{+} \mu^{-}$ pair. The $h^{\prime}$ and $h^{\prime \prime}$ bosons, however, couple in a chiralitychanging way to $\mu^{+} \mu^{-}$. Hence, the helicity combinations of $\mu^{+} \mu^{-}$can only be $\left(h_{+}, h_{-}\right)=\left(\frac{1}{2}, \frac{1}{2}\right)$ and $\left(-\frac{1}{2},-\frac{1}{2}\right)$. Similar considerations apply to $W^{-} \rightarrow \mu^{-} \bar{v}_{\mu}$ versus $H^{-} \rightarrow$ $\mu^{-} \bar{v}_{\mu}$ and $W^{+} \rightarrow \mu^{+} v_{\mu}$ versus $H^{+} \rightarrow \mu^{+} v_{\mu}$.

Two different parameter sets for the Higgs-boson masses are used, see Table 1. For all other particles, PDG recommendations [25] are chosen. For each channel and parameter configuration, at least 200,000 events $(1,000,000$ events) are generated for the signal (background) processes. All simulations are performed for the LHC design energy $\sqrt{s}=14 \mathrm{TeV}$ as well as its current center-of-mass energy $\sqrt{s}=8 \mathrm{TeV}$. 
Table 1 Higgs-boson mass parameter sets used for the comparison of MCPM signatures with SM background. For $h^{\prime}, h^{\prime \prime}$ and $H^{ \pm}$the widths as calculated from Table 3 of [12] are also given. For the mass parameters chosen these widths are practically equal to the total widths. All numbers are in units of $\mathrm{GeV}$

\begin{tabular}{lllllrrr}
\hline Set & $m_{\rho^{\prime}}$ & $m_{h^{\prime}}$ & $\Gamma_{h^{\prime}}$ & $m_{h^{\prime \prime}}$ & $\Gamma_{h^{\prime \prime}}$ & $m_{H^{ \pm}}$ & $\Gamma_{H^{ \pm}}$ \\
\hline A & 125 & 200 & 12.08 & 150 & 9.06 & 150 & 9.07 \\
B & 125 & 400 & 24.16 & 300 & 18.12 & 300 & 18.14 \\
\hline
\end{tabular}

The analysis is first performed without applying any phase-space cuts. Of course, there is no detector that is able to detect and reconstruct every muon. For instance, in the ATLAS detector at CERN the muon trigger chambers only cover the pseudorapidity region $|\eta|<2.4$; see [26]. In addition, selection cuts on the transverse momentum of the muons are applied by the experiments in order to guarantee efficient triggering and background suppression; a typical cut value is $p_{T}>25 \mathrm{GeV}$. Therefore, we also perform the analysis requiring

$|\eta|<2.4$

$p_{T}>25 \mathrm{GeV}$

for each muon. In the $\mu^{-} \bar{v}_{\mu}$ channel,

$E_{T}^{\text {miss }}>25 \mathrm{GeV}$

is also required, where $E_{T}^{\text {miss }}$ is the missing transverse energy due to the non-detection of the neutrino.

For the $\mu^{-} \mu^{+}$channel, the resulting distribution of the invariant mass $m\left(\mu^{-}, \mu^{+}\right)$of the muon pair is shown in Fig. 4 for a center-of-mass energy of $\sqrt{s}=14 \mathrm{TeV}$ and in Fig. 5 for $\sqrt{s}=8 \mathrm{TeV}$. Here and in the following the bin size in mass is chosen as $\Delta m=5 \mathrm{GeV}$. Note that the resonance peaks from the Higgs bosons are enhanced by a factor 10 . Thus, these Higgs-boson resonances are tiny in comparison to the background. The application of selection cuts and a higher center-of-mass energy improve the situation slightly.

For the $\mu^{-} \bar{v}_{\mu}$ channel, the event distribution in the transverse mass $m_{T}$ of the lepton pair is given in Figs. 6 and 7 for $\sqrt{s}=14$ and $8 \mathrm{TeV}$, respectively. Again, the Higgs-boson resonances are much smaller than the background, even using appropriate selection cuts and a center-of-mass energy of $14 \mathrm{TeV}$.

At this point it is appropriate to recall that we have calculated both, the signal and the background processes, to leading order in QCD. Of course, QCD corrections to the SM background processes discussed above are known, but such corrections have not yet been calculated for the production processes of $h^{\prime}, h^{\prime \prime}$, and $H^{ \pm}$in $p p$ collisions. A first estimate of the reactions (22) indicates that they may enhance the Higgs-boson production by something like 20 to $30 \%$. But at this level one also has to consider the virtual

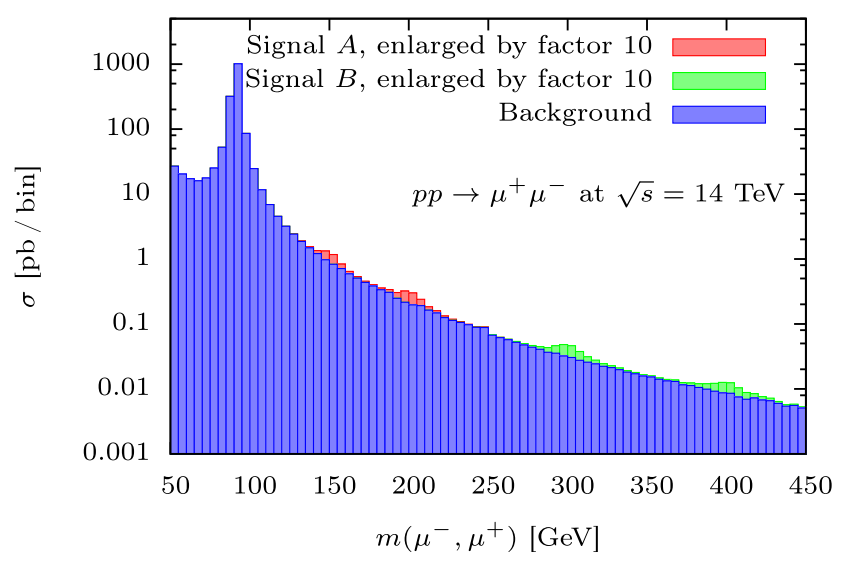

(a)

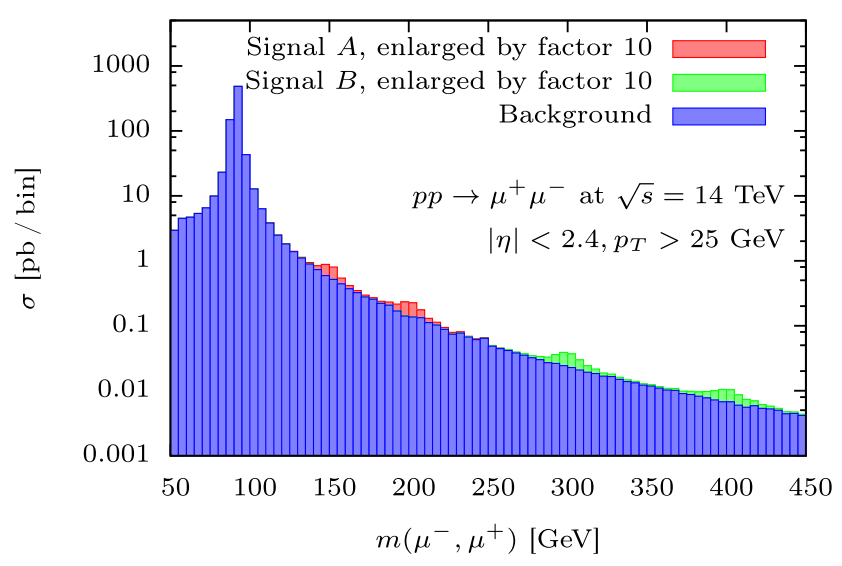

(b)

Fig. 4 MCPM events compared to the SM background in the $\mu^{+} \mu^{-}$ channel as a function of the invariant mass of the muon pair for $p p$ collisions at a center-of-mass energy of $\sqrt{s}=14 \mathrm{TeV}$. Two different parameter sets $A$ and $B$ are used for the MCPM, see Table 1. (a) No selection cuts applied; (b) selection cuts on $\eta$ and $p_{T}$ of the muons applied

corrections to the leading order processes shown in Fig. 1. A complete calculation of $\mathrm{QCD}$ corrections to our processes must be left for future work.

Using the distributions after the application of selection cuts we now calculate, at leading order QCD, how much statistics is needed so that the MCPM resonance peaks are locally significant compared to the background, i.e. larger than $n \sigma$, where $\sigma$ is the statistical uncertainty of the background. Conventionally, an excess with $n=5$ is considered a discovery, while $n=2$ is often used for exclusion limits.

The detector resolution is a limiting factor in the search for resonances. Depending on the channel, the search strategy, the energy scale, the pseudorapidity region and a number of other parameters, the ATLAS collaboration quotes e.g. a design value for the dimuon invariant-mass resolution between 2 and $12 \mathrm{GeV}$; for the transverse-mass resolution the quoted values are slightly worse [26]. Based on these 


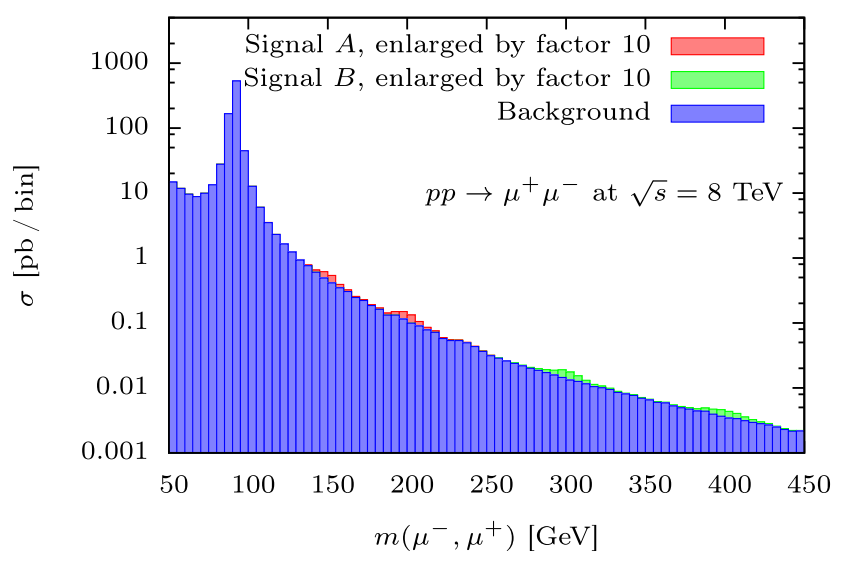

(a)

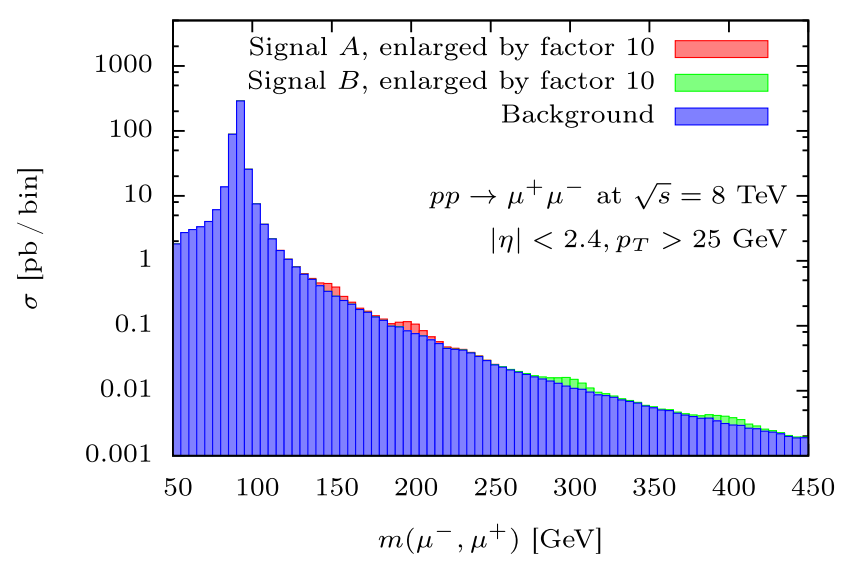

(b)

Fig. 5 Same as Fig. 4, but with a center-of-mass energy of $\sqrt{s}=8 \mathrm{TeV}$. (a) No selection cuts applied; (b) selection cuts on $\eta$ and $p_{T}$ of the muons applied

figures and on the widths $\Gamma$ of the Higgs bosons as given in Table 1, two to five bins were taken into account when calculating the integrated luminosities needed for a local $2 \sigma$ and $5 \sigma$ significance, depending on the mass scale.

In Table 2 we give the integrated luminosities needed for local $2 \sigma$ or $5 \sigma$ significance of the peaks due to the Higgs bosons $h^{\prime}, h^{\prime \prime}$ and $H^{-}$of the MCPM, as shown in Figs. 4b, $5 \mathrm{~b}, 6 \mathrm{~b}$ and $7 \mathrm{~b}$. It should be stressed that these results are very rough estimates. On the one hand, only the local significance in one channel for the selection cuts given in (24a), (24b) is described. The sensitivity of the search might be improved by selection-cut optimisation for the different channels. On the other hand, only hard tree-level processes are taken into account and the behaviour of the detector was not simulated.

Keeping this caveat in mind, these numbers may nevertheless hint at the order of magnitude of statistics needed for MCPM signatures to become visible.

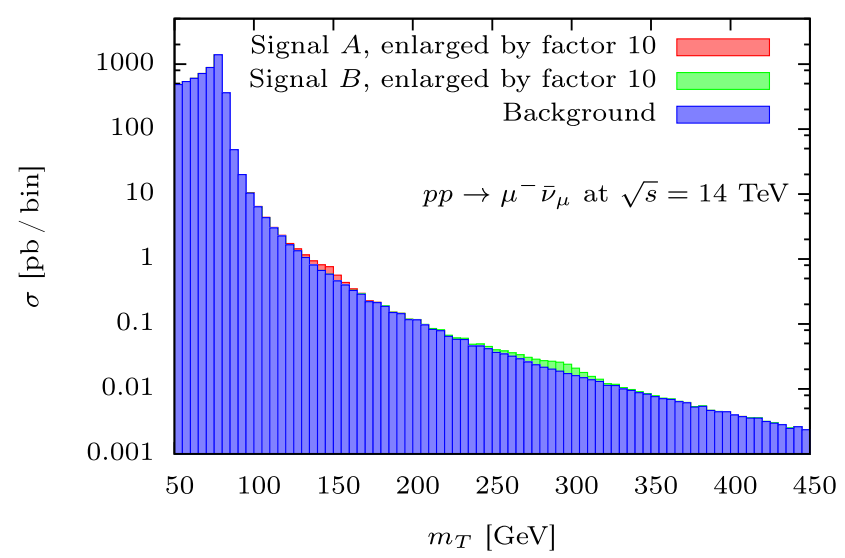

(a)

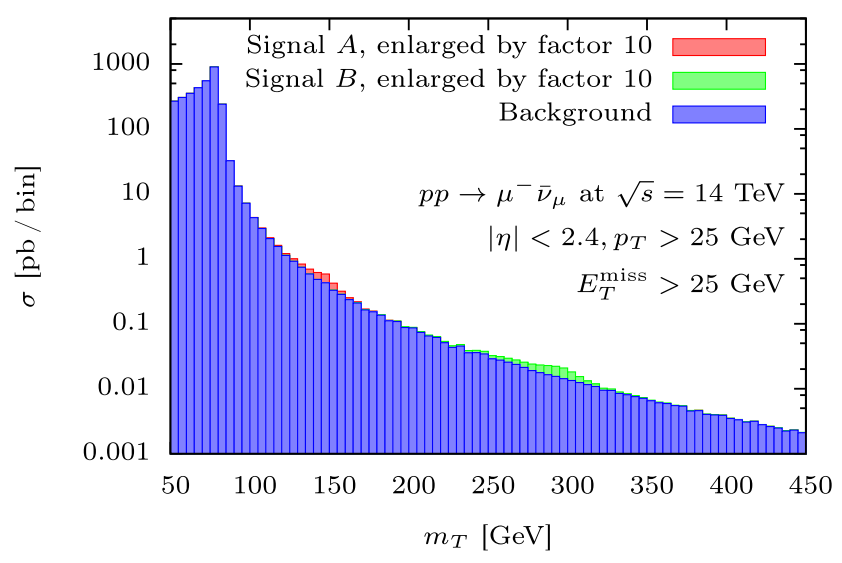

(b)

Fig. 6 MCPM events compared to the SM background in the $\mu^{-} \bar{v}_{\mu}$ channel as a function of the transverse mass of the final state lepton pair for $p p$ collisions at a center-of-mass energy of $\sqrt{s}=14 \mathrm{TeV}$. Again the parameter sets $A$ and $B$ are used for the MCPM, see Table 1. (a) No selection cuts applied; (b) selection cuts on $\eta$ and $p_{T}$ of the muon as well as $E_{T}^{\text {miss }}$ applied

\section{Conclusions}

In this paper we presented the implementation of a twoHiggs-doublet model with maximal CP symmetry, the MCPM, into the Monte Carlo event-generation package MadGraph. To make the paper self contained we recalled in Sects. 1 and 2 the main features of the MCPM. Parts of these chapters are an extended version of results presented at the conference "Physics at LHC 2010" held at DESY in 2010 [14].

The MCPM is an extension of the Standard Model (SM) that is based on the requirement of invariance under certain generalised CP transformations. It features a fermion structure in which only the third-generation fermions are massive. Of course this does not describe nature precisely, but the model gives a first approximation of what has been observed. The theory predicts five physical Higgs bosons. Concerning their Yukawa couplings, four of these bosons couple 


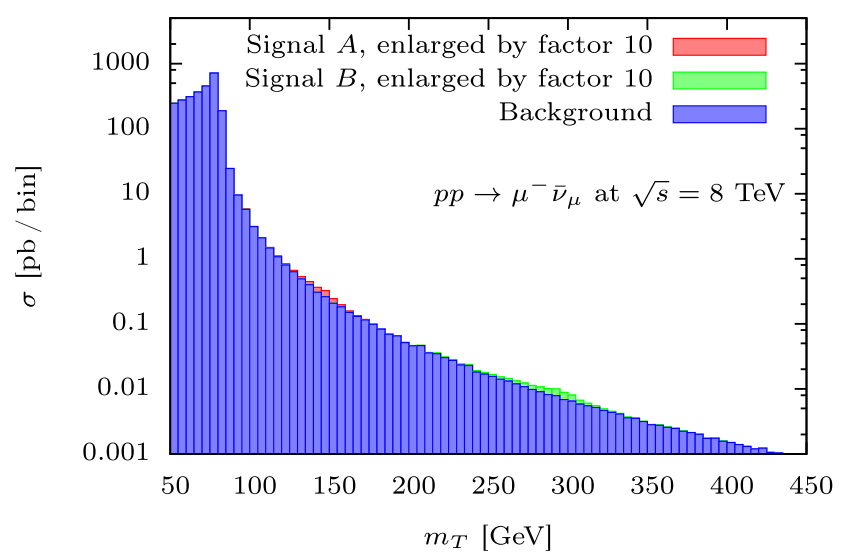

(a)

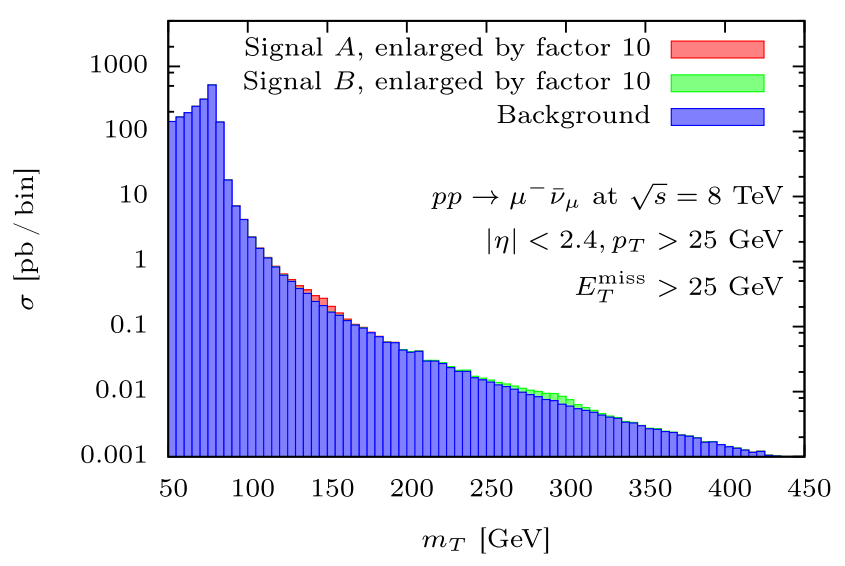

(b)

Fig. 7 Same as Fig. 6, but with a center-of-mass energy of $\sqrt{s}=8 \mathrm{TeV}$. (a) No selection cuts applied; (b) selection cuts on $\eta$ and $p_{T}$ of the muon as well as $E_{T}^{\text {miss }}$ applied only to the second-generation fermions, but with coupling strengths given by the third-generation fermion masses. At colliders such as the LHC, these four Higgs bosons will be produced mainly via quark-antiquark fusion. Their $\mu^{+} \mu^{-}$, $\mu^{+} v_{\mu}$ and $\mu^{-} \bar{v}_{\mu}$ decay modes seem most promising for a discovery. For a proper search for MCPM signatures a Monte Carlo simulation is needed.

We implemented the MCPM into MadGraph, allowing the calculation of cross sections and the random generation of events for arbitrary tree-level processes. The output is in the LHEF format and can be used for further analysis, for instance using PYTHIA and GEANT. Therefore, this MadGraph implementation can be used as the starting point for a full and thorough Monte Carlo simulation of the MCPM. It was validated successfully with different methods. The event generator is available from [27].

The implementation was then used to compare the MCPM signatures to the SM background at LHC energies. This analysis was only done for the muonic channels and restricted to hard processes, so the results are only a rough approximation. For a Higgs boson $h^{\prime \prime}\left(H^{-}\right)$of $300 \mathrm{GeV}$, an integrated luminosity of approximately $100 \mathrm{fb}^{-1}\left(140 \mathrm{fb}^{-1}\right)$ at a center-of-mass energy $\sqrt{s}=8 \mathrm{TeV}$ has to be collected so that a local $2 \sigma$ significance becomes possible. On the other hand, for $h^{\prime \prime}\left(H^{-}\right)$of mass $150 \mathrm{GeV}$ the necessary integrated luminosities at $\sqrt{s}=8 \mathrm{TeV}$ for local $2 \sigma$ significance are only $5.2 \mathrm{fb}^{-1}\left(9.3 \mathrm{fb}^{-1}\right)$. At present (November 2012), the ATLAS and CMS experiments have both collected data representing more than $20 \mathrm{fb}^{-1}$ of integrated luminosity. Thus, it seems that the exclusion of a part of the Higgs-boson mass range or the discovery of small local excesses hinting at the MCPM might be within reach in the near future.
Table 2 Integrated luminosities that are needed for local significances of the Higgs-boson resonances in the invariant mass distribution and transverse mass distribution of the muonic final states $\mu^{+} \mu^{-}$and $\mu^{-} \bar{v}_{\mu}$, respectively. The figures for $\mathrm{H}^{+}$ production and its decay to $\mu^{+} v_{\mu}$ are similar to those for $H^{-}$

\begin{tabular}{|c|c|c|c|c|}
\hline \multirow[t]{2}{*}{ Parameter set } & \multirow[t]{2}{*}{ Process } & \multirow[t]{2}{*}{$m_{H}[\mathrm{GeV}]$} & \multicolumn{2}{|c|}{ Necessary int. luminosity $\int L \mathrm{~d} t\left[\mathrm{fb}^{-1}\right]$} \\
\hline & & & $2 \sigma$ significance & $5 \sigma$ significance \\
\hline \multicolumn{5}{|l|}{ (a) $\sqrt{s}=8 \mathrm{TeV}$} \\
\hline A & $h^{\prime \prime} \rightarrow \mu^{+} \mu^{-}$ & 150 & 5.2 & 33 \\
\hline A & $h^{\prime} \rightarrow \mu^{+} \mu^{-}$ & 200 & 17 & 100 \\
\hline B & $h^{\prime \prime} \rightarrow \mu^{+} \mu^{-}$ & 300 & 99 & 620 \\
\hline B & $h^{\prime} \rightarrow \mu^{+} \mu^{-}$ & 400 & 490 & 3100 \\
\hline A & $H^{-} \rightarrow \mu^{-} \bar{v}_{\mu}$ & 150 & 9.3 & 58 \\
\hline B & $H^{-} \rightarrow \mu^{-} \bar{v}_{\mu}$ & 300 & 140 & 890 \\
\hline \multicolumn{5}{|c|}{ (b) $\sqrt{s}=14 \mathrm{TeV}$} \\
\hline A & $h^{\prime \prime} \rightarrow \mu^{+} \mu^{-}$ & 150 & 1.3 & 8.3 \\
\hline A & $h^{\prime} \rightarrow \mu^{+} \mu^{-}$ & 200 & 3.4 & 21 \\
\hline B & $h^{\prime \prime} \rightarrow \mu^{+} \mu^{-}$ & 300 & 17 & 104 \\
\hline B & $h^{\prime} \rightarrow \mu^{+} \mu^{-}$ & 400 & 64 & 400 \\
\hline A & $H^{-} \rightarrow \mu^{-} \bar{v}_{\mu}$ & 150 & 3.1 & 20 \\
\hline B & $H^{-} \rightarrow \mu^{-} \bar{v}_{\mu}$ & 300 & 30 & 190 \\
\hline
\end{tabular}


Table 3 Feynman rules for the Yukawa couplings of $\rho^{\prime}, h^{\prime}, h^{\prime \prime}$, and $H^{-}$in the MCPM

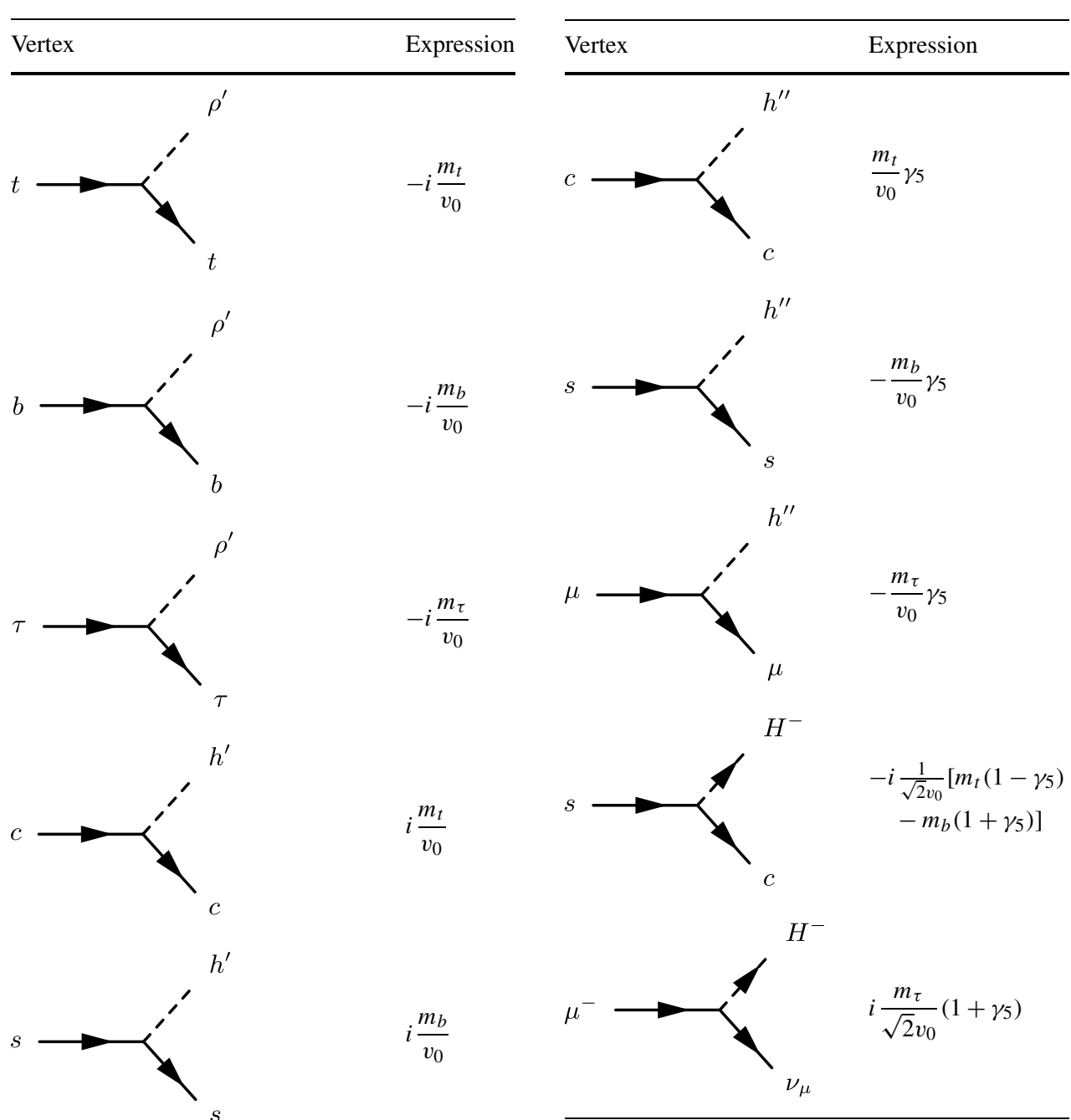

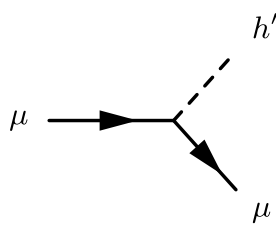

$i \frac{m_{\tau}}{v_{0}}$

Finally, we note that the recent announcement [28, 29] of a signal for a boson of mass $125 \mathrm{GeV}$ is - so far-not only compatible with the SM Higgs boson, but also with the SM-like Higgs boson $\rho^{\prime}$ of the MCPM. Only detailed comparisons of the decay channels of the discovered boson with theoretical predictions will allow to draw further conclusions. We also note that the bounds on the masses of the $h^{\prime}, h^{\prime \prime}$ and $H^{ \pm}$bosons given in [15] depend on the mass of the $\rho^{\prime}$. Identifying the new boson with the $\rho^{\prime}$ of the MCPM, these bounds can be sharpened. Only Fig. 2 of [15] remains relevant where $m_{\rho^{\prime}}=125 \mathrm{GeV}$ was chosen. Our parameter sets $\mathrm{A}$ and $\mathrm{B}$ of Table 1 also correspond to this choice of the $\rho^{\prime}$ mass.
Acknowledgement We want to thank A. von Manteuffel for reading the manuscript and for useful discussions.

Open Access This article is distributed under the terms of the Creative Commons Attribution License which permits any use, distribution, and reproduction in any medium, provided the original author(s) and the source are credited.

\section{Appendix}

\subsection{Feynman rules of the MCPM}

In Appendix A of [12] the MCPM Lagrangian after electroweak symmetry breaking is presented and the Feynman 
rules for several vertices are derived. The most important vertices and the corresponding expressions are given in Table 3 .

\subsection{Using the Monte Carlo generator}

The MCPM model in MadGraph is used in the same way as every MadGraph model, see [16] for an overview. First it has to be loaded. After starting the MadGraph binary in a shell this can be done with the command

import model MCPM -modelname

where the option -model name is needed to ensure the correct particle names. Then processes are defined and the event generation is started:

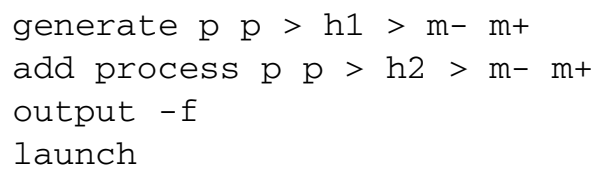

The "generate" and "add process" commands are used to specify the processes MadGraph has to evaluate, while "output" and "launch" start the event generation. The particle names used in the implementation are given in the following section. Before events are produced, MadGraph asks the user whether the standard parameters are to be used or if he wants to modify them.

During the simulation, an in-browser status page keeps the user informed. In the end, MadGraph gives out the contributing Feynman diagrams, the cross-sections or decay widths, the corresponding uncertainty and a compressed file containing the generated events in LHEF [17] format. This can be used for further analysis, for instance with MadAnalysis or PYTHIA [18].

\subsection{MCPM parameter relations}

Here we give the relation — at tree level—between the original parameters of the MCPM Lagrangian and the parameters of (23) used in the event generator. With $v_{0}$ given in terms of the original parameters in (17) we have

$\alpha=\frac{1}{4 \pi} \frac{g^{2} g^{\prime 2}}{g^{2}+g^{\prime 2}}$,

$G_{F}=\frac{1}{\sqrt{2}} v_{0}^{-2}$,

$\alpha_{s}=\frac{g_{s}^{2}}{4 \pi}$,

$m_{Z}=\frac{1}{2} v_{0} \sqrt{g^{2}+g^{\prime 2}}$,

$m_{\tau}=c_{l 3}^{(1)} \frac{v_{0}}{\sqrt{2}}$, $m_{t}=c_{u 3}^{(1)} \frac{v_{0}}{\sqrt{2}}$,

$m_{b}=c_{d 3}^{(1)} \frac{v_{0}}{\sqrt{2}}$,

$m_{\rho^{\prime}}^{2}=2 v_{0}^{2}\left(\eta_{00}+\mu_{3}\right)$,

$m_{h^{\prime}}^{2}=2 v_{0}^{2}\left(\mu_{1}-\mu_{3}\right)$,

$m_{h^{\prime \prime}}^{2}=2 v_{0}^{2}\left(\mu_{2}-\mu_{3}\right)$,

$m_{H^{ \pm}}^{2}=2 v_{0}^{2}\left(-\mu_{3}\right)$.

7.4 List of particles and parameters in the MadGraph implementation of the MCPM

A list of MCPM particles and parameters with their MadGraph names are presented in Tables 4 and 5, respectively.

The parameters may be a bit confusing, because the $c$, $e, \mu$ can be set to be massive, while the $u, d$ and $s$ are always massless. This does not have anything to do with the MCPM. As explained in Sect. 3, some of the consequences of strict symmetry under generalised CP transformations have been dropped in this implementation, so it is generally possible to set masses for the first- and secondgeneration fermions. However, MadGraph treats the very light fermions as massless (even in the SM, where the theory certainly predicts something else). This does not change the physics at collider experiments in the $\mathrm{TeV}$ range, but it saves some computation time. Therefore the list of parameters includes the masses of those fermions of the first two generations where the masses are relevant for the calculations.

MadGraph also allows to set decay widths for all particles, which are named similarly to the mass parameters. This is important for further analysis with programs such as PYTHIA; these parameters do not influence the generation of events with MadGraph at all.

Table 4 The MCPM particles and their names in the MadGraph implementation

\begin{tabular}{ll}
\hline Particles & Names in MadGraph MCPM implementation \\
\hline Protons & $\mathrm{p}$ \\
Quarks & $\mathrm{u} \mathrm{d} \mathrm{C} \mathrm{s} \mathrm{t} \mathrm{b}$ \\
Antiquarks & $\mathrm{u} \sim \mathrm{d} \sim \mathrm{c} \sim \mathrm{s} \sim \mathrm{t} \sim \mathrm{b} \sim$ \\
Leptons & $\mathrm{e}-\mathrm{m}-\mathrm{tt}-\mathrm{ve} \mathrm{vm} \mathrm{vt}$ \\
Antileptons & $\mathrm{e}+\mathrm{m}+\mathrm{tt}+\mathrm{ve} \sim \mathrm{vm} \sim \mathrm{vt} \sim$ \\
Gauge bosons & $\mathrm{a} \mathrm{z} \mathrm{w}+\mathrm{w}-\mathrm{g}$ \\
$\rho^{\prime}, h^{\prime}, h^{\prime \prime}$ & $\mathrm{rho} \mathrm{h} 1 \mathrm{~h} 2$ \\
$H^{ \pm}$ & $\mathrm{h}+\mathrm{h}-$ \\
\hline
\end{tabular}


Table 5 The MCPM parameters and their names in the MadGraph implementation. Note that some parameters are zero in the MCPM (see last column), however they can be set to other values in this model. This allows the correct simulation of SM background processes (see Sect. 3). The CKM matrix is given in the Wolfenstein parametrisation [30]

\begin{tabular}{|c|c|c|c|c|c|}
\hline Section & Parameter & MadGraph & Default & Unit & $\begin{array}{l}\text { Strict } \\
\text { MCPM }\end{array}$ \\
\hline \multirow[t]{4}{*}{ Gauge } & $\frac{1}{\alpha}$ & aEWM1 & 127.916 & & \\
\hline & $G_{F}$ & Gf & 0.000011664 & $\mathrm{GeV}^{-2}$ & \\
\hline & $\alpha_{s}$ & $\mathrm{aS}$ & 0.1184 & & \\
\hline & $m_{Z}$ & MZ & 91.1876 & $\mathrm{GeV}$ & \\
\hline \multirow[t]{4}{*}{ CKM matrix } & $\lambda_{\mathrm{WS}}$ & lamWS & 0.2253 & & 0 \\
\hline & $A_{\mathrm{WS}}$ & AWS & 0.808 & & \\
\hline & $\rho_{\mathrm{WS}}$ & rhows & 0.132 & & \\
\hline & $\eta_{\mathrm{ws}}$ & etaWs & 0.341 & & \\
\hline \multirow{6}{*}{$\begin{array}{l}\text { Fermion } \\
\text { masses }\end{array}$} & $m_{c}$ & $\mathrm{MC}$ & 1.42 & $\mathrm{GeV}$ & 0 \\
\hline & $m_{t}$ & MT & 172.9 & $\mathrm{GeV}$ & \\
\hline & $m_{b}$ & MB & 4.67 & $\mathrm{GeV}$ & \\
\hline & $m_{e}$ & $\mathrm{Me}$ & 0.00051100 & $\mathrm{GeV}$ & 0 \\
\hline & $m_{\mu}$ & MM & 0.10566 & $\mathrm{GeV}$ & 0 \\
\hline & $m_{\tau}$ & MTA & 1.7768 & $\mathrm{GeV}$ & \\
\hline \multirow{4}{*}{$\begin{array}{l}\text { Higgs-boson } \\
\text { masses }\end{array}$} & $m_{\rho^{\prime}}$ & mrho & 125 & $\mathrm{GeV}$ & \\
\hline & $m_{h^{\prime}}$ & $\operatorname{mh} 1$ & 200 & $\mathrm{GeV}$ & \\
\hline & $m_{h^{\prime \prime}}$ & $\operatorname{mh} 2$ & 150 & $\mathrm{GeV}$ & \\
\hline & $m_{H^{ \pm}}$ & mhe & 150 & $\mathrm{GeV}$ & \\
\hline
\end{tabular}

\section{References}

1. J.F. Gunion, H.E. Haber, G.L. Kane, S. Dawson, The Higgs hunter's guide. Front. Phys. 80, 1-448 (2000)

2. H.E. Haber, R. Hempfling, The renormalization group improved Higgs sector of the minimal supersymmetric model. Phys. Rev. D 48, 4280-4309 (1993)

3. F. Nagel, New aspects of gauge-boson couplings and the Higgs sector. PhD thesis, University of Heidelberg, 2004. http://www. ub.uni-heidelberg.de/archiv/4803/

4. M. Maniatis, A. von Manteuffel, O. Nachtmann, F. Nagel, Stability and symmetry breaking in the general two-Higgs-doublet model. Eur. Phys. J. C 48, 805-823 (2006)

5. C.C. Nishi, CP violation conditions in $N$-Higgs-doublet potentials. Phys. Rev. D 74, 036003 (2006)

6. M. Maniatis, A. von Manteuffel, O. Nachtmann, CP violation in the general two-Higgs-doublet model: a geometric view. Eur. Phys. J. C 57, 719-738 (2008)

7. T.D. Lee, G.C. Wick, Space inversion, time reversal, and other discrete symmetries in local field theories. Phys. Rev. 148, 13851404 (1966)

8. G. Ecker, W. Grimus, W. Konetschny, Quark mass matrices in leftright symmetric gauge theories. Nucl. Phys. B 191, 465 (1981)

9. G. Ecker, W. Grimus, H. Neufeld, Spontaneous CP violation in left-right symmetric gauge theories. Nucl. Phys. B 247, 70-82 (1984)
10. P.M. Ferreira, H.E. Haber, M. Maniatis, O. Nachtmann, J.P. Silva, The geometric picture of generalized-CP and Higgs-family transformations in the two-Higgs-doublet model. Int. J. Mod. Phys. A 26, 769-808 (2011)

11. M. Maniatis, A. von Manteuffel, O. Nachtmann, A new type of CP symmetry, family replication and fermion mass hierarchies. Eur. Phys. J. C 57, 739-762 (2008)

12. M. Maniatis, O. Nachtmann, On the phenomenology of a twoHiggs-doublet model with maximal CP symmetry at the LHC. J. High Energy Phys. 0905, 028 (2009)

13. M. Maniatis, O. Nachtmann, On the phenomenology of a twoHiggs-doublet model with maximal CP symmetry at the LHC, II: radiative effects. J. High Energy Phys. 1004, 027 (2010)

14. M. Maniatis, O. Nachtmann, A. von Manteuffel, On the phenomenology of a two-Higgs-doublet model with maximal $\mathrm{CP}$ symmetry at the LHC: synopsis and addendum, in Proceedings of the Fifth Conference on Physics at the LHC (2010), DESY-PROC2010-01. The general permission to draw on the articles of these proceedings as stated there is acknowledged

15. M. Maniatis, O. Nachtmann, Symmetries and renormalisation in two-Higgs-doublet models. J. High Energy Phys. 1111, 151 (2011)

16. J. Alwall, M. Herquet, F. Maltoni, O. Mattelaer, T. Stelzer, MadGraph 5: going beyond. J. High Energy Phys. 1106, 128 (2011)

17. J. Alwall, A. Ballestrero, P. Bartalini, S. Belov, E. Boos et al., A standard format for Les Houches event files. Comput. Phys. Commun. 176, 300-304 (2007)

18. T. Sjostrand, P. Eden, C. Friberg, L. Lonnblad, G. Miu et al., Highenergy physics event generation with PYTHIA 6.1. Comput. Phys. Commun. 135, 238-259 (2001)

19. C. Degrande, C. Duhr, B. Fuks, D. Grellscheid, O. Mattelaer et al., UFO - the universal FeynRules output. Comput. Phys. Commun. 183, 1201-1214 (2012)

20. J. Brehmer, Monte-Carlo event generation for a two-Higgsdoublet model with maximal CP symmetry (2012)

21. ATLAS Collaboration, Search for dilepton resonances in pp collisions at $\sqrt{s}=7 \mathrm{TeV}$ with the ATLAS detector. Phys. Rev. Lett. 107, 272002 (2011)

22. CMS Collaboration, Search for narrow resonances in dilepton mass spectra in pp collisions at $\sqrt{s}=7 \mathrm{TeV}$. Phys. Lett. B 714, 158-179 (2012)

23. ATLAS Collaboration, Search for a heavy gauge boson decaying to a charged lepton and a neutrino in $1 \mathrm{fb}^{-1}$ of pp collisions at $\sqrt{s}=7 \mathrm{TeV}$ using the ATLAS detector. Phys. Lett. B 705, 28-46 (2011)

24. CMS Collaboration, Search for leptonic decays of $\mathrm{W}^{\prime}$ bosons in $\mathrm{pp}$ collisions at $\sqrt{s}=7$ TeV. J. High Energy Phys. 1208, 023 (2012)

25. K. Nakamura et al., Review of particle physics. J. Phys. G 37, 075021 (2010)

26. ATLAS Collaboration, ATLAS detector and physics performance. Technical Design Report, 1. CERN, Geneva, 1999

27. J. Brehmer, MCPM model files for MadGraph 5 (2012). http://cp3. irmp.ucl.ac.be/projects/madgraph/wiki/Models/MCPM

28. ATLAS Collaboration, Observation of a new particle in the search for the Standard Model Higgs boson with the ATLAS detector at the LHC. Phys. Lett. B 716, 1-29 (2012)

29. CMS Collaboration, Observation of a new boson at a mass of $125 \mathrm{GeV}$ with the CMS experiment at the LHC. Phys. Lett. B 716, 30-61 (2012)

30. L. Wolfenstein, Parametrization of the Kobayashi-Maskawa matrix. Phys. Rev. Lett. 51, 1945 (1983) 